\title{
Blood glucose concentration for predicting poor outcomes in patients with and without impaired glucose metabolism undergoing off-pump coronary artery bypass surgery - long-term observational study
}

\author{
Wojciech Szychta ${ }^{1}$, Franciszek Majstrak², Grzegorz Opolski ${ }^{1}$, Krzysztof J. Filipiak $^{1}$ \\ ${ }^{1} 1^{\text {st }}$ Chair and Department of Cardiology, Medical University of Warsaw, Warsaw, Poland \\ ${ }^{2}$ Department of Cardiac Surgery, $1^{\text {st }}$ Chair of Cardiology, Medical University of Warsaw, Warsaw, Poland
}

Adv Interv Cardiol 2016; 12, 3 (45): 238-246

DOI: 10.5114/aic.2016.61646

\begin{abstract}
Introduction: Strict glucose control is an everyday practice in the perioperative period. Elevated glucose level has a deleterious impact on clinical results, but a therapeutic target has not been stated yet.

Aim: To determine a glucose concentration range affecting long-term outcomes after coronary artery bypass surgery (CABG).

Material and methods: This study is a retrospective evaluation of consecutive patients treated in a university hospital in Poland from 2004 to 2008. Patients were divided into 2 groups: an impaired glucose metabolism group (IGM) if they had 1) known DM or 2) perioperative hyperglycaemia defined as $\geq 200 \mathrm{mg} / \mathrm{dl}$; and a non-IGM group. The end point (EP) was all-cause mortality.

Results: One thousand two hundred and eleven patients were covered by the analysis. The observation time was from 01.01.2004 until 01.08.2012. Patients who had maximal glucose concentrations < $242 \mathrm{mg} / \mathrm{dl}$ had the lowest mortality risk (EP in $21.1 \%$ ); a higher risk was noted in the group with glucose concentrations $242-324 \mathrm{mg} / \mathrm{dl}$ (EP in $30.8 \%$ ); and a very high risk was found for the group where glucose concentration was $>324 \mathrm{mg} / \mathrm{dl}(\mathrm{EP}$ in $44.2 \%)(p=0.041)$. Patients with IGM had a shorter survival at the end of the study $(p<0.001)$. The longest survival was observed in patients whose maximal glucose level was $\leq 242 \mathrm{mg} /$ $\mathrm{dl}(p<0.001)$ and the minimal glucose concentration was in the range $61-110 \mathrm{mg} / \mathrm{dl}(p<0.001)$.

Conclusions: Tight glucose concentration control should be performed irrespective of a diabetes diagnosis and proper treatment introduced when necessary. Maximal glucose concentration should be kept $<242 \mathrm{mg} / \mathrm{dl}$, while the minimum should be in the range $60-110 \mathrm{mg} / \mathrm{dl}$.
\end{abstract}

Key words: diabetes, concentration, glucose, mortality, off-pump coronary artery bypass surgery, off-pump.

\section{Introduction}

Diabetes mellitus (DM) is a risk factor for coronary artery disease (CAD) and a common co-morbidity, which can be found in up to $40.4 \%$ of cases referred for cardiac surgery [1]. Some patients have DM diagnosed after having the first symptoms of CAD. The disease often coexists with advanced, diffused CAD [2] and has an impact on multiorgan failure, which worsens the prognosis [3-5]. Patients in this group benefit mostly from surgical revascularisation [6]. Current European Society of Cardiology (ESC) guidelines support screening for DM in CAD patients, but clinical follow-up in the postoperative period is still under discussion [6].
Strict glucose control is an everyday practice in perioperative period as it improves outcome [7]. Elevated glucose level has an aggravating impact on clinical results, although a therapeutic target has not been stated yet [3, 6-9]. The first studies revealed reduction by $3 \%$ of mortality in patients treated aggressively with insulin, having the target serum glucose of $80-110 \mathrm{mg} / \mathrm{dl}$ in comparison to the conventional target of $180-200 \mathrm{mg} / \mathrm{dl}$ [10]. Those results were not confirmed in a study with three target groups -1$)$ tight ( $\leq 126 \mathrm{mg} / \mathrm{dl}), 2)$ moderate $(127-179$ $\mathrm{mg} / \mathrm{dl})$ and 3) liberal ( $\geq 180 \mathrm{mg} / \mathrm{dl})$ glucose control. The moderate group had the lowest mortality $(2.9 \%$ vs. moderate $2.0 \%$ vs. liberal $3.4 \%$ ) and incidence of major complications (19.4\% vs. moderate $11.1 \%$ vs. liberate $14.2 \%$ ) [7].

\section{Corresponding author:}

Wojciech Szychta MD, PhD, $1^{\text {st }}$ Chair and Department of Cardiology, Medical University of Warsaw, 1a Banacha St, 01-049 Warsaw, Poland, phone: +48 2259919 58, fax: +48 2259919 57, e-mail: wszychta@wp.pl

Received: 18.10.2015, accepted: 15.12.2015 
Further research (NICE-SUGAR trial) proved a $2.8 \%$ reduction of mortality in a group of patients treated with standard $(<180 \mathrm{mg} / \mathrm{dl})$ in comparison to relative and tight control of glucose concentration $(81-108 \mathrm{mg} / \mathrm{dl})$ in patients admitted to the intensive care unit (ICU) who were expected to require treatment on $\geq 3$ consecutive days [11].

\section{Aim}

The aim of the study was to determine a glucose concentration range affecting long-term outcomes after offpump coronary artery bypass surgery (OPCAB).

\section{Material and methods}

\section{Study population}

This study is a retrospective, observational evaluation of successive patients treated in a university hospital in Poland from 2004 to 2008. The analysis was conducted on data collected from medical records of patients. Inclusion criteria were: $C A D$ patients with surgical indication for OPCAB through median sternotomy and the same surgeon. Exclusion criteria were: combined procedures (operations different from isolated OPCAB) and conversion to surgery with cardiopulmonary bypass.

Patients were divided into 2 groups: an impaired glucose metabolism group (IGM) if they had 1) known DM or 2) perioperative hyperglycaemia defined as $\geq 200 \mathrm{mg} /$ $\mathrm{dl}$, and a non-IGM group in other cases. Such criteria were set to create a group of patients with $\mathrm{DM}$, poorly controlled or undiagnosed DM and stress-induced insulin resistance, as about $50 \%$ of patients are believed to be undiagnosed for presence of DM in the preoperative period [6]. The perioperative period was defined as from the day of surgery until discharge from the ICU. Patients were treated according to ESC guidelines [6] for patients with $D M$ referred for coronary artery bypass surgery (CABG). Data on mortality were collected from the Ministry of Internal Affairs National Civil Register.

Maximal and minimal concentrations of serum glucose were analysed. Hypoglycaemia was defined as a serum glucose level of $<60 \mathrm{mg} / \mathrm{dl}$ and severe hypoglycaemia was defined as a serum glucose level of $\leq 40 \mathrm{mg} / \mathrm{dl}$. Anaemia was defined as a haemoglobin level $<12 \mathrm{~g} / \mathrm{dl}$ in women and $<13.5 \mathrm{~g} / \mathrm{dl}$ in men. Past smoking was defined as quitting at least 2 weeks before surgery. Such criteria were set as the first 2 weeks are critical in determining quitting failure rates [12]. The 'accelerated' mode was in fact elective surgery; however, patients in this group were admitted to the Department of Cardiac Surgery directly from another clinic/cardiology ward without being discharged from a referring hospital. We have introduced the term 'logistic EuroSCORE', which is the predicted mortality according to the logistic regression equation.

This paper is part of a larger study. We are also monitoring long-term outcomes after OPCAB, and the first results have already been reported elsewhere $[13,14]$.

\section{End point}

The end point was all-cause mortality.

\section{Statistical analysis}

The results are presented as mean \pm standard deviation for numerical data. For categorical data, results are shown as a percentage. The $\chi^{2}$ test of independence was used for comparison of the frequency of occurrence of immeasurable data in the analysed subgroups. Analysis of variance (ANOVA) was used to compare the frequency of numerical data in the analysed subgroups. Decision exhaustive $\chi^{2}$ automatic interaction detection (CHAID) trees were performed to transform the numerical variables to compartment variables, maximizing the variability of death/survival analysis. The survival was visualized using Kaplan-Meier graphs.

In case of maximal glycaemia, the analysis was conducted after verification of histograms and unusual observations (deviating beyond the third standard deviation) and then removed from the database. Such a statistical procedure resulted in inclusion of $1207 \mathrm{pa}$ tients for this parameter. Statistical significance was determined at the level of $p<0.05$. Statistical analyses were carried out using IBM SPSS, Excel and Latex.

\section{Matching procedure}

A set of covariates was selected to estimate the propensity score. These were: age, body mass index (BMI), neurological dysfunction affecting activity, occurrence of peripheral artery disease and EuroSCORE. The propensity score matched (PSM) group of patients was selected using logistic regression to evaluate predicted probability of belonging to a group. The result was interpreted as a 'balance vector' for further matching. Finally, IGM patients were matched with non-IGM controls using the nearest neighbour technique in a ratio of $1: 1$.

Finally, we performed log-rank analysis to verify the impact of IGM on lifetime expectancy.

\section{Results}

We identified 1248 patients out of a total population of 2739. As some data were missing, we included 1211 patients ( 896 men $-73.99 \%$ ) aged $65.6 \pm 9.47$ years who met the inclusion criteria in the study. The observation continued from 01.01.2004 until 01.08.2012. At the end of the observation period, 903 patients were alive $(74.56 \%)$, while 308 (25.43\%) had reached the end point (Figure 1). All variables were well balanced with the matching procedure; the standardized differences of means between covariates can be seen in Figure 2 . After the matching procedure, 'logistic EuroSCORE' was the only parameter which significantly differentiated the two groups. 


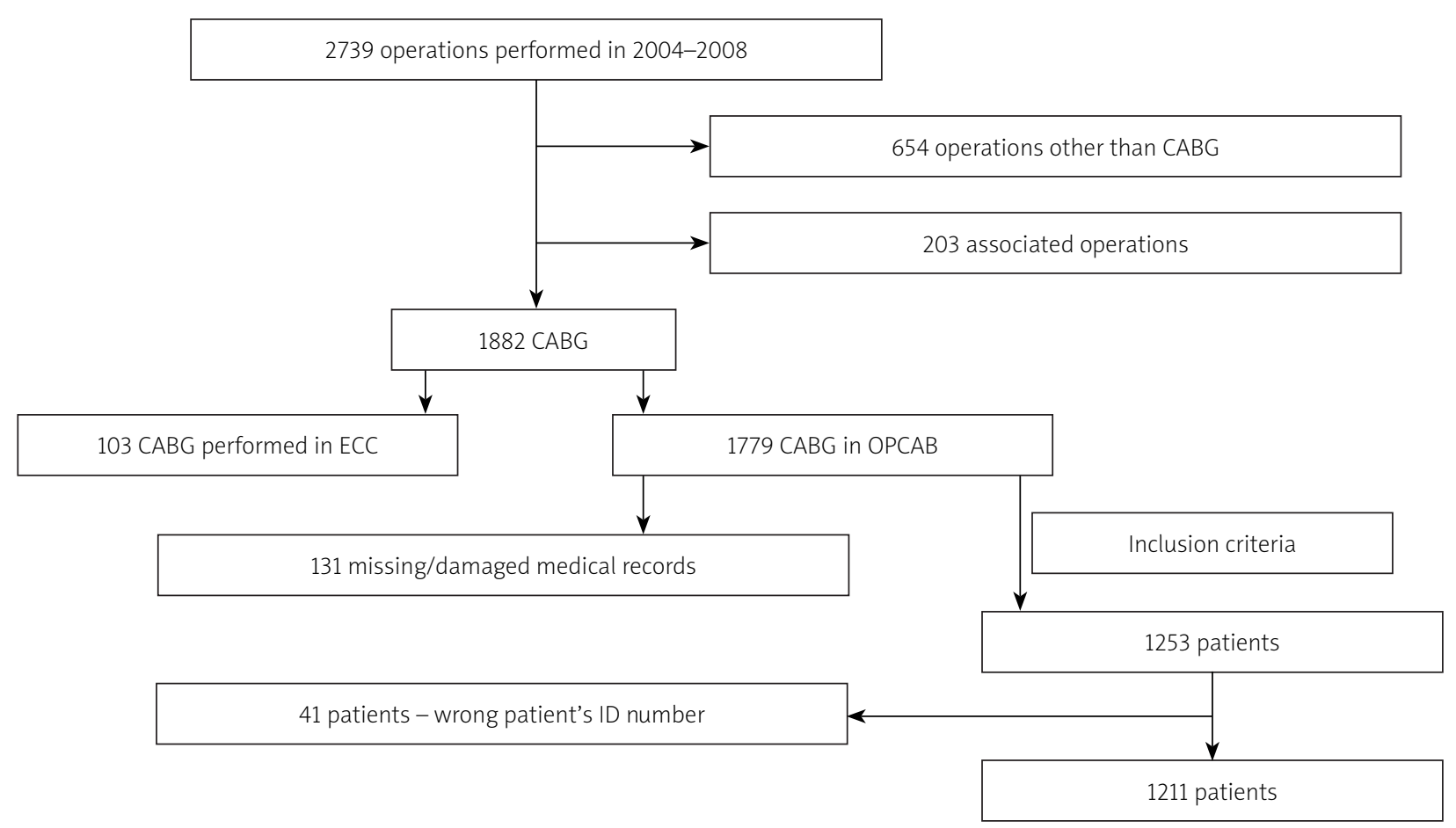

Figure 1. Flow chart showing longitudinal analysis of the study population
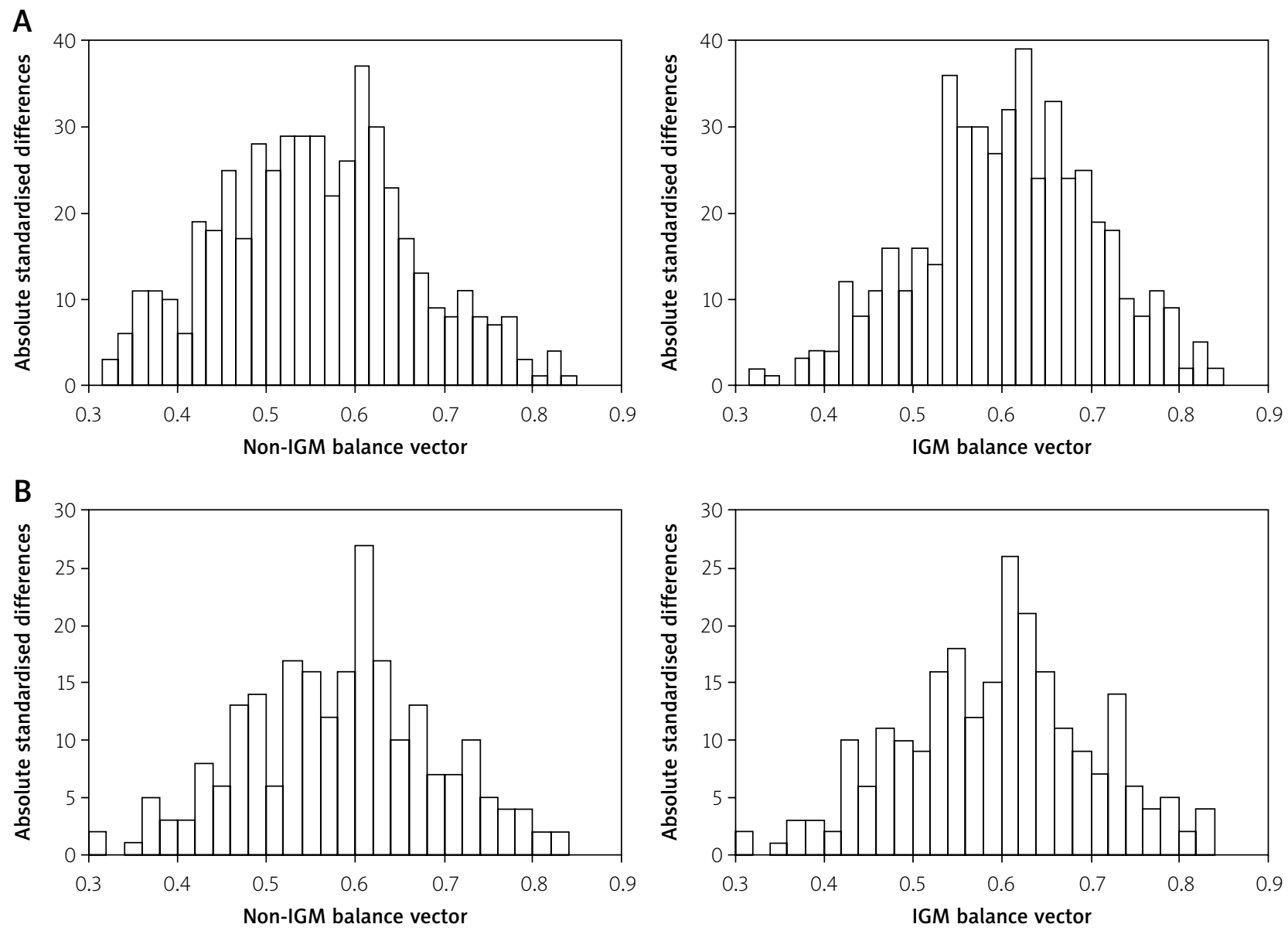

Figure 2. Distributions of balance vector for both groups: A - before matching procedure, B - after matching procedure 


\section{Characteristics of patients}

We identified 731 (58.6\%) IGM patients, aged 66.58 \pm 9.09 years. Patients with IGM were significantly more often: women, older, with a higher body mass index (BMI). The IGM group had more frequently coexisting: hypertension, extracardiac arteriopathy and neurological dysfunction affecting their activity. However, they less commonly had previous percutaneous coronary intervention (PCI) (Table I). No significant differences were found between the groups when analysed for left ventricular ejection fraction (EF) and severity of CAD (Table II).

Patients with IGM had a higher risk of mortality (logistic and additive EuroSCORE). Venous grafts were more often were implanted during the surgery, but right internal mammary (RIMA) grafts are less common than in the non-IGM group. No differences between the groups were found when frequency of using the left internal mammary artery (LIMA) as a graft was compared. In the postoperative period, the IGM group incurred a higher risk of such complications as resternotomy, atrial fibrillation (AF) in ICU, need for intra-aortic balloon pump (IABP) and sudden cardiac death (SCD), especially in the mechanism of ventricular fibrillation and tachycardia (VF/VT), and suffered from respiratory failure. Those patients had significantly more units of packed red blood cells (PRBC) transfused (Tables I,

Table I. Population characteristics

\begin{tabular}{|c|c|c|c|c|c|c|}
\hline \multirow[t]{2}{*}{ Parameter } & \multicolumn{3}{|c|}{ Before propensity matching } & \multicolumn{3}{|c|}{ After propensity matching } \\
\hline & IGM (707) & Non-IGM (504) & $P$-value & IGM (243) & Non-IGM (230) & $P$-value \\
\hline Sex (women) & $209(29.6 \%)$ & $106(21 \%)$ & 0.01 & $66(27.2 \%)$ & $50(21.7 \%)$ & 0.171 \\
\hline Sex (men) & $498(70.4 \%)$ & $398(79 \%)$ & & $177(72.8 \%)$ & $180(78.3 \%)$ & \\
\hline Age [years] & $66.59 \pm 8.86$ & $64.11 \pm 10.07$ & $<0.0001$ & $66.16 \pm 9.03$ & $65.73 \pm 9.95$ & 0.623 \\
\hline $\mathrm{BMI}\left[\mathrm{kg} / \mathrm{m}^{2}\right]$ & $28.44 \pm 4.47$ & $27.32 \pm 3.87$ & $<0.0001$ & $28.07 \pm 4.58$ & $28.08 \pm 3.83$ & 0.984 \\
\hline Hypertension & $573(81 \%)$ & $362(71.8 \%)$ & $<0.001$ & $191(78.6 \%)$ & $173(75.2 \%)$ & 0.382 \\
\hline GRF $\left[\mathrm{ml} / \mathrm{min} / 1.73 \mathrm{~m}^{2}\right]$ & $70.06 \pm 21.92$ & $75.83 \pm 22.49$ & 0.801 & $69.17 \pm 21.75$ & $73.25 \pm 21.05$ & 0.039 \\
\hline Active tobacco smoking & $144(20.4 \%)$ & $119(23.6 \%)$ & 0.177 & $61(25.1 \%)$ & $46(20 \%)$ & 0.185 \\
\hline History of previous smoking & $296(41.9 \%)$ & $217(43.1 \%)$ & 0.68 & $95(39.1 \%)$ & $102(44.3 \%)$ & 0.247 \\
\hline ACS $<90$ days & $232(32.8 \%)$ & $178(35.3 \%)$ & 0.364 & $85(35 \%)$ & $85(37 \%)$ & 0.654 \\
\hline History of previous CABG & $10(0.8 \%)$ & $5(0.4 \%)$ & 0.512 & $1(0.4 \%)$ & $3(1.3 \%)$ & 0.289 \\
\hline Anaemia & $218(30.8 \%)$ & $138(27.4 \%)$ & 0.193 & $76(31.3 \%)$ & $75(32.3 \%)$ & 0.756 \\
\hline CPD & $82(11.6 \%)$ & $51(10.1 \%)$ & 0.417 & $30(12.3 \%)$ & $19(8.3 \%)$ & 0.145 \\
\hline Neurological dysfunction & $85(12 \%)$ & $33(6.5 \%)$ & 0.002 & $26(10.7 \%)$ & $16(7 \%)$ & 0.153 \\
\hline Extracardiac arteriopathy & $207(29.3 \%)$ & $112(22.2 \%)$ & 0.006 & $69(28.4 \%)$ & $62(27 \%)$ & 0.727 \\
\hline Preoperative PCl & $96(13.6 \%)$ & $91(18.1 \%)$ & 0.034 & $36(14.8 \%)$ & $38(16.5 \%)$ & 0.61 \\
\hline Preoperative $\mathrm{PCl}<3$ months & $32(4.5 \%)$ & $39(7.8 \%)$ & 0.019 & $18(7.4 \%)$ & $17(7.4 \%)$ & 0.995 \\
\hline Additive EuroSCORE & $5.42 \pm 3.50$ & $4.69 \pm 3.25$ & $<0.0001$ & $5.47 \pm 3.72$ & $5.09 \pm 3.19$ & 0.232 \\
\hline Logistic EuroSCORE (\%) & $7.92 \pm 10.80$ & $6.01 \pm 7.80$ & 0.001 & $8.38 \pm 11.7$ & $6.48 \pm 7.59$ & 0.038 \\
\hline Hospitalization [days] & $17.87 \pm 19.28$ & $14.99 \pm 8.21$ & 0.001 & $17.32 \pm 14.27$ & $15.7 \pm 8.42$ & 0.137 \\
\hline ICU [days] & $6.33 \pm 4.83$ & $5.23 \pm 2.16$ & $<0.0001$ & $6.39 \pm 4.88$ & $5.28 \pm 2.26$ & 0.002 \\
\hline
\end{tabular}

ACS - acute coronary syndrome, BMI - body mass index, CPD - chronic pulmonary diseases, GFR - glomerular filtration rate, IGM - impaired glucose metabolism, $\mathrm{PCl}$ - percutaneous coronary intervention, ICU - intensive care unit.

Table II. Cardiovascular parameters on admission day

\begin{tabular}{|c|c|c|c|c|c|c|c|}
\hline \multirow{2}{*}{\multicolumn{2}{|c|}{ Parameter }} & \multicolumn{3}{|c|}{ Before propensity matching } & \multicolumn{3}{|c|}{ After propensity matching } \\
\hline & & IGM (707) & Non-IGM (504) & $P$-value & IGM (243) & Non-IGM (230) & $P$-value \\
\hline \multicolumn{2}{|c|}{$E F>50 \%$} & $391(55.3 \%)$ & $272(54 \%)$ & 0.645 & $139(57.2 \%)$ & $121(52.6 \%)$ & 0.316 \\
\hline \multicolumn{2}{|c|}{$E F=30-50 \%$} & $288(40.7 \%)$ & $216(42.9 \%)$ & 0.46 & $94(38.7 \%)$ & $108(47 \%)$ & 0.069 \\
\hline \multicolumn{2}{|c|}{$E F<30 \%$} & $32(4.5 \%)$ & $20(4.0 \%)$ & 0.637 & $12(4.9 \%)$ & $4(1.7 \%)$ & 0.054 \\
\hline \multirow[t]{3}{*}{ CAD } & Single-vessel & $120(17 \%)$ & $95(18.9 \%)$ & 0.201 & $41(16.9 \%)$ & $38(16.5 \%)$ & 0.889 \\
\hline & Two-vessel & $323(45.7 \%)$ & $245(48.7 \%)$ & & $112(46.1 \%)$ & $111(48.3 \%)$ & \\
\hline & Three-vessel & $264(37.3 \%)$ & $163(32.4 \%)$ & & $90(37 \%)$ & $81(35.2 \%)$ & \\
\hline
\end{tabular}

$C A D$ - coronary artery disease, IGM - impaired glucose metabolism, EF-left ventricular ejection fraction. 
III, IV). Hypoglycaemia was found in 57 (4.7\%) patients and severe hypoglycaemia in 20 (1.65\%) patients of the study population.

The IGM group stayed longer in the ICU, followed by a longer hospitalization (Table I). On discharge, patients with IGM were less often administered: acetylsalicylic acid, $\beta$-blockers, angiotensin-converting enzyme (ACE) inhibitors and statins. However, significantly more IGM patients had administered sartans and calcium channel blockers (Table V).

Propensity score matched patients from the IGM group stayed longer in the ICU. Moreover, in the post- operative period, the IGM group still incurred a higher risk of any complication, especially resternotomy, $A F$ in $I C U$, need for IABP and SCD, more often in the mechanism of VF/VT, and suffered from respiratory failure. These patients also had significantly more units of PRBC transfused. Patients from the PSM IGM group on discharge were less often administered ACE inhibitors and statins. However, significantly more of them were administered sartans (Tables I, IV, V). Finally, they had shorter mean lifetime duration than respondents from the non-IGM group (age: 79.31 vs. 87.18 years; $\chi^{2}=6.14$; $p=0.013)$.

Table III. Intraoperative parameters

\begin{tabular}{|c|c|c|c|c|c|c|c|}
\hline \multicolumn{2}{|c|}{ Parameter } & \multicolumn{3}{|c|}{ Before propensity matching } & \multicolumn{3}{|c|}{ After propensity matching } \\
\hline & & IGM (707) & Non-IGM (504) & $P$-value & IGM (243) & Non-IGM (230) & $P$-value \\
\hline \multirow[t]{3}{*}{ CAD } & Stable & $496(70.2 \%)$ & $363(72 \%)$ & 0.079 & $165(67.9 \%)$ & $170(73.9 \%)$ & 0.057 \\
\hline & Unstable & $118(16.7 \%)$ & $95(18.8 \%)$ & & $42(17.3 \%)$ & $42(18.3 \%)$ & \\
\hline & ACS & $93(13.2 \%)$ & $46(9.1 \%)$ & & $36(14.8 \%)$ & $18(7.8 \%)$ & \\
\hline \multicolumn{2}{|c|}{ Elective } & $389(55.0 \%)$ & $292(57.9 \%)$ & 0.486 & $129(53.1 \%)$ & $131(57 \%)$ & 0.449 \\
\hline \multicolumn{2}{|c|}{ Accelerated } & $208(29.4 \%)$ & $149(29.6 \%)$ & & $73(30 \%)$ & $71(30.9 \%)$ & \\
\hline \multicolumn{2}{|c|}{ Urgent } & $80(11.3 \%)$ & $47(9.3 \%)$ & & $29(11.9 \%)$ & $22(9.6 \%)$ & \\
\hline \multicolumn{2}{|c|}{ Emergency } & $30(4.2 \%)$ & $16(3.2 \%)$ & & $12(4.9 \%)$ & $6(2.6 \%)$ & \\
\hline \multicolumn{2}{|c|}{ Venous grafts } & $498(70.4 \%)$ & $318(63.1 \%)$ & 0.007 & $168(69.1 \%)$ & $156(67.8)$ & 0.759 \\
\hline \multicolumn{2}{|c|}{ LIMA grafts } & $683(96.6 \%)$ & $496(95.6 \%)$ & 0.384 & $236(97.1 \%)$ & $219(95.2 \%)$ & 0.28 \\
\hline \multicolumn{2}{|c|}{ RIMA grafts } & $122(17.3 \%)$ & $111(22 \%)$ & 0.033 & $43(17.7 \%)$ & $43(18.7 \%)$ & 0.778 \\
\hline \multicolumn{2}{|c|}{ LRA grafts } & $30(4.2 \%)$ & $28(5.6 \%)$ & 0.292 & $9(3.7 \%)$ & $10(4.3 \%)$ & 0.721 \\
\hline \multicolumn{2}{|c|}{ Anastomosis } & $2.34 \pm 0.92$ & $2.28 \pm 0.95$ & 0.859 & $2.34 \pm 0.91$ & $2.34 \pm 0.92$ & 0.936 \\
\hline \multicolumn{2}{|c|}{ Operation [min] } & $179.07 \pm 53.99$ & $172.98 \pm 52.89$ & 0.866 & $178.8 \pm 54.84$ & $172.74 \pm 52.9$ & 0.223 \\
\hline
\end{tabular}

ACS - acute coronary syndrome, CAD - coronary artery disease, IGM - impaired glucose metabolism, LIMA - left internal mammary artery, RIMA - right internal mammary artery, LRA - left radial artery.

Table IV. Perioperative variables

\begin{tabular}{lcccccc} 
& \multicolumn{3}{c}{ Pefore propensity matching } & \multicolumn{3}{c}{ After propensity matching } \\
\cline { 2 - 7 } & IGM (707) & Non-IGM (504) & $P$-value & IGM (243) & Non-IGM (230) & $P$-value \\
\hline Chest tube drainage $[\mathrm{ml}]$ & $708.59 \pm 406.9$ & $714.85 \pm 366.11$ & 0.981 & $692.2 \pm 367.1$ & $729.6 \pm 403.02$ & 0.292 \\
\hline PRBC $[\mathrm{U}]$ & $2.47 \pm 3.08$ & $1.7 \pm 1.8$ & $<0.0001$ & $2.7 \pm 3.5$ & $1.75 \pm 1.9$ & 0.0003 \\
\hline Any complication & $186(26.3 \%)$ & $110(21.8 \%)$ & 0.074 & $68(28 \%)$ & $44(19.1 \%)$ & 0.024 \\
\hline Resternotomy & $59(8.3 \%)$ & $13(2.6 \%)$ & $<0.0001$ & $26(10.7 \%)$ & $7(3 \%)$ & 0.001 \\
\hline In-hospital infections & $94(13.3 \%)$ & $71(14.1 \%)$ & 0.725 & $29(11.9 \%)$ & $31(13.5 \%)$ & 0.614 \\
\hline IABP & $65(9.2 \%)$ & $10(2.0 \%)$ & $<0.001$ & $28(11.5 \%)$ & $3(1.3 \%)$ & $<0.001$ \\
\hline AF in ICU & $310(43.8 \%)$ & $151(30.0 \%)$ & $<0.001$ & $99(40.7 \%)$ & $70(30.4 \%)$ & 0.019 \\
\hline SCD & $26(3.7 \%)$ & $3(0.6 \%)$ & 0.001 & $11(4.5 \%)$ & 0 & 0.001 \\
\hline VF/VT & $19(2.7 \%)$ & $4(0.8 \%)$ & 0.017 & $6(2.5 \%)$ & 0 & 0.016 \\
\hline Asystole/PEA & $5(0.7 \%)$ & $0(0 \%)$ & 0.059 & $3(1.2 \%)$ & 0 & 0.091 \\
\hline Stroke & $5(0.7 \%)$ & $2(0.4 \%)$ & 0.482 & $2(0.8 \%)$ & 0 & 0.168 \\
\hline Dialysis & $18(2.5 \%)$ & $3(0.6 \%)$ & $<0.0001$ & $5(2.1 \%)$ & $1(0.4 \%)$ & 0.115 \\
\hline Respiratory failure & $25(3.5 \%)$ & $4(0.8 \%)$ & $<0.0001$ & $9(3.7 \%)$ & $2(0.9 \%)$ & 0.041
\end{tabular}

AF - atrial fibrillation, IGM - impaired glucose metabolism, IABP - intra-aortic balloon pump, ICU - postoperative intensive cardiac unit, PEA - pulseless electrical activity, PRBC - packed red blood cells, SCD - sudden cardiac death, VF-ventricular fibrillation, VT - ventricular tachycardia. 
Table V. Pharmacological treatment administered after coronary artery bypass grafting (CABG)

\begin{tabular}{lcccccc} 
& \multicolumn{3}{c}{ Gefore propensity matching } & \multicolumn{3}{c}{ After propensity matching } \\
\cline { 2 - 7 } & IGM (707) & Non-IGM (504) & $P$-value & IGM (243) & Non-IGM (230) & $P$-value \\
\hline ASA & $629(89.0 \%)$ & $480(95.2 \%)$ & $<0.001$ & $216(88.9 \%)$ & $216(93.9 \%)$ & 0.052 \\
\hline$\beta$-blocker & $646(91.4 \%)$ & $483(95.8 \%)$ & 0.002 & $226(93 \%)$ & $220(95.7 \%)$ & 0.215 \\
\hline ACE inhibitor & $566(80.1 \%)$ & $433(85.9 \%)$ & 0.008 & $194(79.8 \%)$ & $204(88.7 \%)$ & 0.008 \\
\hline Sartan & $33(2.8 \%)$ & $4(1.0 \%)$ & 0.027 & $9(3.7 \%)$ & $1(0.4 \%)$ & 0.014 \\
\hline Statin & $607(85.9 \%)$ & $474(94.0 \%)$ & $<0.001$ & $210(86.4 \%)$ & $217(94.3 \%)$ & 0.004 \\
\hline Ca-blocker & $129(18.2 \%)$ & $67(13.3 \%)$ & 0.021 & $48(19.8 \%)$ & $36(15.7 \%)$ & 0.243 \\
\hline Diuretic & $575(81.3 \%)$ & $413(81.9 \%)$ & 0.786 & $198(81.5 \%)$ & $198(86.1 \%)$ & 0.175 \\
\hline Aldosterone antagonist & $32(4.5 \%)$ & $18(3.6 \%)$ & 0.41 & $13(5.3 \%)$ & $6(2.6 \%)$ & 0.129 \\
\hline LMWH & $213(30.1 \%)$ & $166(32.9 \%)$ & 0.299 & $76(31.3 \%)$ & $68(29.6 \%)$ & 0.686 \\
\hline Metformin & $70(9.9 \%)$ & & & $24(9.9 \%)$ & &
\end{tabular}

ACE-angiotensin-converting enzyme, ASA - acetylsalicylic acid, Ca-blocker-calcium channel blocker, IGM-impaired glucose metabolism, LMWH - low molecular weight heparin.

\section{Mortality risk according to glucose concentration}

Analysis was based on the decision exhaustive CHAID tree. Patients who had a maximal glucose concentration $<242 \mathrm{mg} / \mathrm{dl}$ had the lowest mortality risk (21.1\% reached the end point). A higher risk (30.8\% reached the end point) was noted in the group with glucose concentrations 242$324 \mathrm{mg} / \mathrm{dl}$, and a very high risk was found for the group where the glucose concentration was $>324 \mathrm{mg} / \mathrm{dl}(44.2 \%$ reached the end point) $\left(\chi^{2}=34.22 ; p=0.041\right)$.

Using log-rank tests, we found that patients with IGM had shorter survival at the end of the study $(M=79.42$; $S E=1.35$ vs. $M=86.47$ in months, respectively; $S E=$ $1.35 ; \chi^{2}=12.33 ; p<0.001$ ) (Figure 3). Moreover, the longest survival time was found in patients whose maximal glucose level was $\leq 242 \mathrm{mg} / \mathrm{dl}\left(\chi^{2}=10.73 ; p<0.001\right)$, a shorter lifetime than in the first group was observed among patients whose the glucose concentration was within 242-324 mg/dl $\left(\chi^{2}=29.82 ; p<0.001\right)$, and the shortest survival was found when glucose concentration exceeded $324 \mathrm{mg} / \mathrm{dl}\left(\chi^{2}=4.45 ; p<0.001\right)(M=85.55$; $S E=1.08$ vs. $M=78.00 ; S E=2.35$ vs. $M=68.83 ; S E=$ 3.69 in months, respectively) (Table VI, Figure 4). Minimal glucose concentration determined the shortest survival time when the glucose level was $\leq 60 \mathrm{mg} / \mathrm{dl}(p<0.001)$, a longer survival was found among patients whose minimal glucose level was $>110 \mathrm{mg} / \mathrm{dl}(p=0.03)$, but the longest survival time was found in the group of patients whose minimal glucose concentration was in the range 61-110 mg/dl $(p<0.001)(M=59.05 ; S E=6.36$ vs. $M=77.16 ; S E=3.34$ vs. $M=83.93 ; S E=1.1$ in months, respectively) (Table VI, Figure 5).

\section{Discussion}

One of the main results of this study is that we determined the range of blood glucose concentration related to poor long-term outcomes. Our results were influenced by treatment method, as all patients were operated on without cardiopulmonary bypass. Such a technique allows for better control of glucose concentration, as it has a negative impact on postoperative glycaemia both in the DM and the non-DM population [15]. We analysed glucose concentrations during hospitalisation in the ICU, though previous research showed that a glucose concentration $\geq 158 \mathrm{mg} / \mathrm{dl}$ on the first postoperative day is associated with death, in-hospital infections and prolonged stay in the ICU [16]. On the other hand, patients who have $\geq 3$ hypoglycaemic episodes $(<74 \mathrm{mg} / \mathrm{dl})$ also suffered from increased mortality and complications [16].

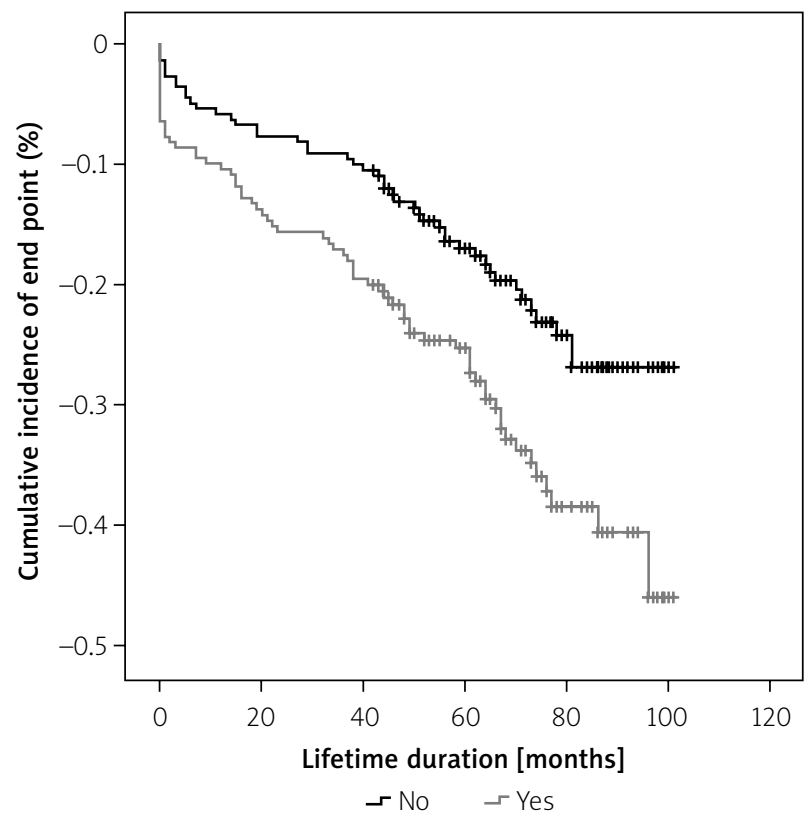

Figure 3. Postoperative survival in patients with IGM and without IGM undergoing OPCAB in the long-term follow-up: propensity scored analysis 
Table VI. Mortality risk according to impaired glucose metabolism (IGM) occurrence and glucose concentration based on decision exhaustive $\chi^{2}$ automatic interaction detection (CHAID) tree analysis and theoretical groups in Cox regression models for survival time

\begin{tabular}{|c|c|c|c|c|}
\hline \multirow[t]{2}{*}{ Parameter } & \multirow[t]{2}{*}{$P$-value } & \multirow[t]{2}{*}{ OR } & \multicolumn{2}{|c|}{$\mathrm{Cl} 95 \%$ for OR } \\
\hline & & & Lower limit & Upper limit \\
\hline IGM & $<0.01$ & 1.52 & 1.20 & 1.93 \\
\hline Maximal glycaemia - categories based on CHAID decision trees $\leq 242 \mathrm{mg} / \mathrm{dl}$ & $<0.01$ & & & \\
\hline Maximal glycaemia - categories based on CHAID decision trees $242-324 \mathrm{mg} / \mathrm{dl}$ & $<0.01$ & 1.56 & 1.19 & 2.05 \\
\hline Maximal glycaemia - categories based on CHAID decision trees > 324 mg/dl & $<0.01$ & 2.29 & 1.68 & 3.12 \\
\hline Minimal glycaemia - theoretical category $<60$ mg/dl & $<0.01$ & & & \\
\hline Minimal glycaemia - theoretical category $61-110$ mg/dl & $<0.01$ & 0.35 & 0.23 & 0.55 \\
\hline Minimal glycaemia - theoretical category > $110 \mathrm{mg} / \mathrm{dl}$ & 0.03 & 0.55 & 0.32 & 0.93 \\
\hline
\end{tabular}

Cl-confidence interval, OR - odds ratio.

To our knowledge, this is one of the first studies with a long-term follow-up. Previous studies were focused on in-hospital mortality [16, 17], 30-day mortality [5, 7, 15, 17] and 90-day mortality [11]. According to our results, the highest mortality can be seen in the early period of observation, although Kaplan-Meier graphs show a deteriorating prognosis for patients with a maximal glucose concentration $>242 \mathrm{mg} / \mathrm{dl}$ and minimal glycaemia $>110 \mathrm{mg} /$ dl or $<60 \mathrm{mg} / \mathrm{dl}$.

Current ESC guidelines do not indicate a target glucose concentration in the postoperative period, but only refer to the results of a study showing that the glucose concentration should be kept in the range $120-180 \mathrm{mg} / \mathrm{dl}$ by insulin infusions as such a protocol is followed by best outcomes in comparison to more intensive and less intensive glucose management $[6,7]$. However, the Society

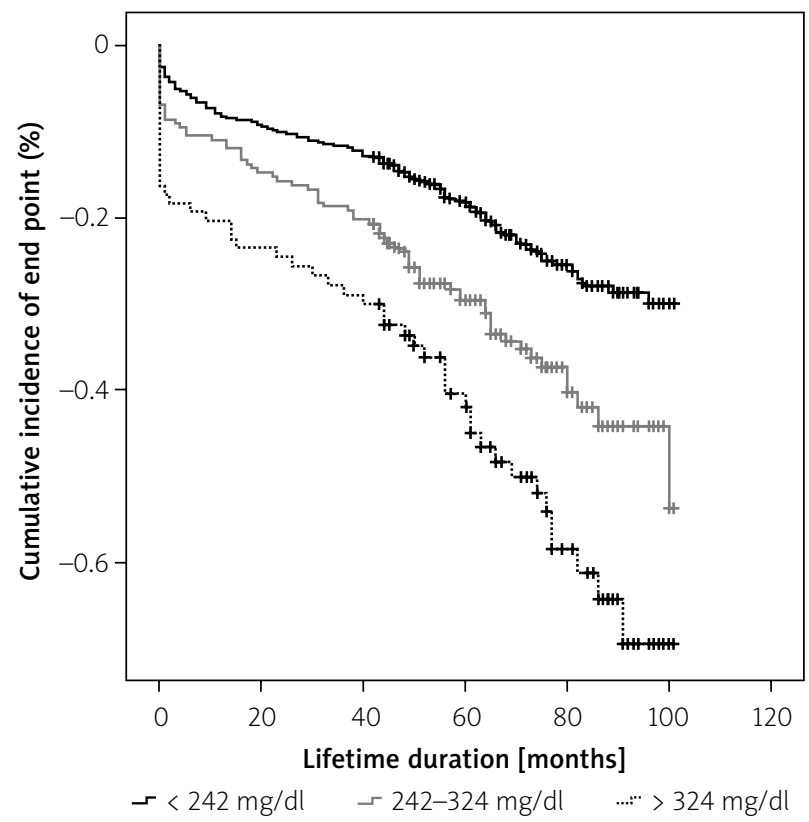

Figure 4. Postoperative survival in patients according to maximum glucose concentration groups - based on CHAID decision trees of Thoracic Surgeons (STS) supports the recommendation that all patients with persistently elevated serum glucose (>180 mg/dl) should receive intravenous insulin infusions to maintain their glucose concentration < $180 \mathrm{mg} / \mathrm{dl}$ for the duration of their ICU care, and this should also be the target glucose level in the peak postprandial state. Also patients who require $\geq 3$ days in the ICU due to respiratory complications or unstable hemodynamic condition, regardless of the diabetic status, should be treated with intravenous insulin injections to achieve glucose concentrations $<150 \mathrm{mg} / \mathrm{dl}$. What is more, the STS recommends reaching a blood glucose level $\leq 110$ $\mathrm{mg} / \mathrm{dl}$ in fasting and pre-meal states in further follow-up [18]. However, a meta-analysis of 29 randomized trials, considering 8432 patients, reported no significant differences in mortality when compared to the treatment tar-

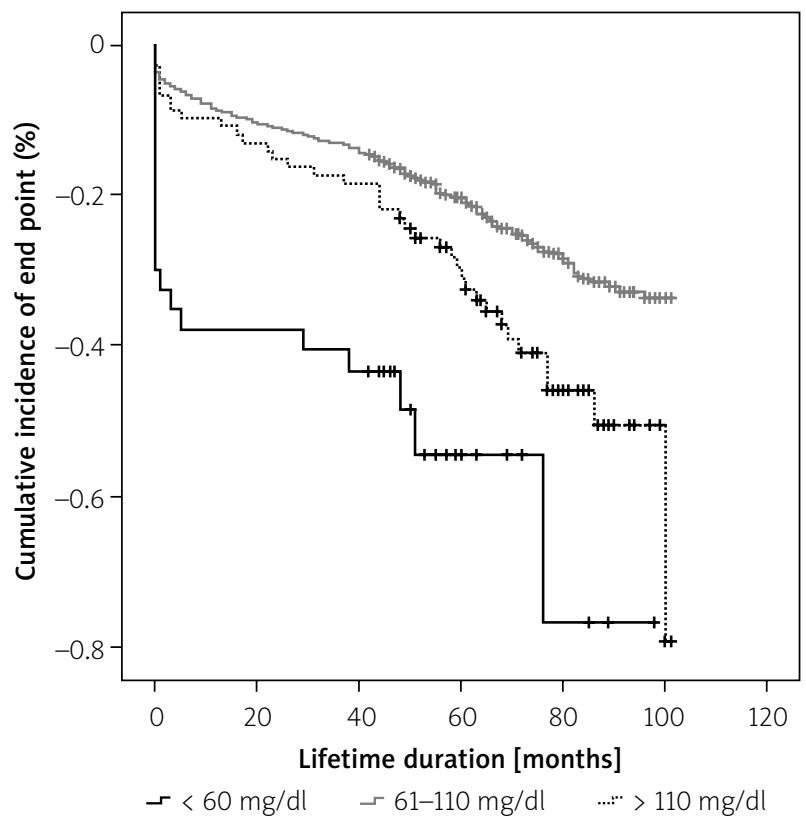

Figure 5. Postoperative survival in patients with minimal glucose concentration - based on theoretical categories 
get of $\leq 110 \mathrm{mg} / \mathrm{dl}$ vs. $<150 \mathrm{mg} / \mathrm{dl}$, having a higher risk of severe hypoglycaemia (defined by glucose concentrations $\leq 40 \mathrm{mg} / \mathrm{dl}$ ) [17]. In our institution, we apply a protocol to maintain glucose concentrations $<180 \mathrm{mg} / \mathrm{dl}$. Our results show clinical difficulties in everyday clinical practice. The highest glucose concentration was usually found on the first day after surgery. Unfortunately, we did not analyse time since leaving the operating theatre and the highest glucose level.

The IGM population analysed in our group was similar to those previously described. Studies considering patients with DM in the perioperative setting of cardiac surgery report that those patients are more often: older [2], women [2, 3, 19], with a higher BMI [2, 3, 19]. Those patients have more often coexisting: hypertension [2, 3, 19], dyslipidaemia [2], renal insufficiency, peripheral artery disease [2, 5] and anaemia [2]. Patients with DM are less often in New York Heart Association class I-II [2, 19] and have more often depressed $\operatorname{EF}[5,19]$. However, we are aware that there are reports stating that non-DM patients are older [19], more often active smokers [2], and chronic pulmonary diseases can be more frequently diagnosed in that group [5], and they less often undergo $\mathrm{PCl}$ before the CABG [19]. We have also found that those patients more commonly suffer from neurological disorders affecting their everyday activity and have a coexisting extracardiac arteriopathy.

In an operative setting, patients with DM usually require more anastomosis. Some authors report a longer operative time [3], but we did not observe such a correlation. However, we found that patients with IGM had less often used RIMA, which was reported previously [2], yet this tendency is not universal [19]. In our study, although there was no difference in grafting LIMA between the analyzed groups, the IGM group more often received the greater saphenous vein (SVG) as a graft. That approach is related to a usually diffused CAD, which makes it technically difficult for a surgeon to achieve complete revascularization using only arterial grafts. Unfortunately, 12 years after CABG, 55\% of SVG are patent, $18 \%$ narrowed and $26 \%$ are closed, whereas $96 \%$ of RIMA and LIMA grafts are patent and less than $4 \%$ closed [20].

The IGM group more readily suffers from renal complications, postoperative bleeding [3] and infections $[5,19]$ in the postoperative period. We also found that IGM patients are more likely to have onsets of AF in ICU. Moreover, we found that these patients are more likely to require resternotomy due to excessive postoperative bleeding and transfusions of PRBC. Although we did not collect information on indications for IABP implantation, IGM patients were more often supported with IABP. These observations are followed by both a longer hospitalization in the ICU and in-hospital stays, which is corroborated by another study [19].

We have found one study where no difference in pharmacological administration between DM and no-DM groups was found, although the possibilities of medical treatment (MT) were still underused (respectively, DM vs. no-DM: aspirin $95 \%$ vs. $94 \%$; $\beta$-blockers $80 \%$ vs. $79 \%$; ACE inhibitor/sartans $71 \%$ vs. $60 \%$; statin $90 \%$ vs. $91 \%$ ) [4]. The IGM patients operated on in our unit had significantly underused possibilities of pharmacotherapy in comparison to the non-IGM group. This could result in poorer clinical outcomes, which was found in the longterm follow-up. However, we have no data verifying whether our patients' MT changed over time in outpatient clinics.

We found that the estimated mortality risk (both in logistic and additive EuroSCORE) was higher in the IGM group, which was clinically confirmed by more frequent SCD in ICU. Even after a successful matching procedure, logistic EuroSCORE was the only parameter which significantly differentiated the two groups. However, DM is one of the factors increasing the EuroSCORE value itself. On the other hand, authors from the US calculated that patients from non-DM groups had a higher estimated mortality risk [19]. Previous reports show that in-hospital mortality decreased in the DM group over time (from $2.7 \%$ in 1992 to $0.7 \%$ in 2001 [2]; from $3.1 \%$ in $1998-$ 2002 to $1 \%$ in 2003-2005 [19]). Such an evaluation is confirmed by short-term observational studies, where no differences were observed in the non-DM population in terms of mortality $[3,5]$. However, when the end point was analyzed as all-cause deaths, overall infection and acute renal failure, the DM group of patients had a significantly worse outcome [5]. In the long-term follow-up, mortality risk was higher for patients with DM and stable CAD than in the no-DM group, regardless of the treatment option ( $\mathrm{MT}, \mathrm{PCl}, \mathrm{CABG})$ [4]. Subjects undergoing $C A B G$ had the lowest risk of cardiac mortality in a 10-year follow-up (respectively MT, PCI, CABG: $26.1 \%$ vs. $18.8 \%$ vs. $12.5 \%$; $p=0.005$ for CABG vs. MT, others $p>0.05$ ) [4]. A study involving 39.235 patients concluded that the mortality risk is two times higher in patients with DM type 1, whereas for type 2 it increased only slightly. Diabetes mellitus type 1 is usually a longer lasting disease (DM type $1-40.8$ vs. DM type $2-9.6$ years; $94 \%$ of DM type 1 had $>20$ years of disease vs. $10 \%$ of DM type 2) [21]. In our study, we observed an impact of the disease on a patient's prognosis. This results not only from proven clinical factors but also from the clinical profile of patients, protocol of surgery (use of arterial grafts) and postoperative pharmacological treatment [20, 22]. Type of DM could have very little impact on the results of the study as only 2 patients had DM type 1 diagnosed.

There are several limitations to this study. This was a retrospective, observational project. The blood tests could be masked by a catecholamine-mediated physiological response to hypoglycaemia, which is non-excludable in an ICU setting. Our sample was large, but not large enough to stratify the level of glucose concentration control as a marker for risk events, as we still had too 
few complications. Blood glucose concentrations taken into account were measured in the hospital's laboratory, but also in an ICU device used for emergency cases. Patients analysed in the study not only had stable CAD, but some of them were operated with the diagnosis of acute coronary syndrome or, on admission, were in a critical clinical condition. We did not collect information on international names of drugs or drug doses, but we were aiming to administer the highest tolerated doses.

\section{Conclusions}

Patients with IGM have shorter lifetime duration. Moreover, they are burdened with more risk factors, which further worsens the prognosis after OPCAB. These patients have a higher risk of complications, especially resternotomy, AF in ICU, in-hospital infections and SCD. The possibilities of pharmacotherapy in diabetic patients are underused. Glucose concentration should be closely monitored irrespective of diagnosed diabetes and proper treatment introduced when necessary. The maximal glucose concentration should be kept $<242 \mathrm{mg} / \mathrm{dl}$, while the minimum should be in the range $60-110 \mathrm{mg} / \mathrm{dl}$.

\section{Conflict of interest}

The authors declare no conflict of interest.

\section{References}

1. ElBardissi AW, Aranki SF, Sheng S, et al. Trends in isolated coronary artery bypass grafting: an analysis of the Society of Thoracic Surgeons adult cardiac surgery database. J Thorac Cardiovasc Surg 2012; 143: 273-81.

2. Antunes PE, de Oliveira JF, Antunes MJ. Coronary surgery in patients with diabetes mellitus: a risk-adjusted study on early outcome. Eur J Cardiothorac Surg 2008; 34: 370-5.

3. Koochemeshki V, Salmanzadeh H, Sayyadi H, et al. The effect of diabetes mellitus on short term mortality and morbidity after isolated coronary artery bypass grafting surgery. Int Cardiovasc Res J 2013; 7: 41-5.

4. Lima EG, Hueb W, Garcia RM, et al. Impact of diabetes on 10year outcomes of patients with multivessel coronary artery disease in the Medicine, Angioplasty, or Surgery Study II (MASS II) trial. Am Heart J 2013; 166: 250-7.

5. Minakata K, Bando K, Takanashi S, et al. Impact of diabetes mellitus on outcomes in Japanese patients undergoing coronary artery bypass grafting. J Cardiol 2012; 59: 275-84.

6. Rydén L, Grant PJ, Anker SD, et al. ESC Guidelines on diabetes, pre-diabetes, and cardiovascular diseases developed in collaboration with the EASD. Eur Heart J 2013; 34: 3035-87.

7. Bhamidipati CM, LaPar DJ, Stukenborg GJ, et al. Superiority of moderate control of hyperglycemia to tight control in patients undergoing coronary artery bypass grafting. J Thorac Cardiovasc Surg 2011; 141: 543-51.

8. Fujii T, Watanabe Y, Shiono N, et al. Usefulness of perioperative blood glucose control in patients undergoing off-pump coronary artery bypass grafting. Gen Thorac Cardiovasc Surg 2007; 55: 409-15.
9. Lazar HL, McDonnell MM, Chipkin S, et al. Effects of aggressive versus moderate glycemic control on clinical outcomes in diabetic coronary artery bypass graft patients. Ann Surg 2011; 254: 458-63; discussion 463-4.

10. Van den Berghe G, Wouters P, Weekers F, et al. Intensive insulin therapy in critically ill patients. N Engl J Med 2001; 345: 1359-67.

11. Finfer S, Chittock D, Su S, et al. Intensive versus conventional glucose control in critically ill patients. N Engl I Med 2009; 360: 1283-97.

12. Kenford $S$, Fiore $M$, Jorenby $D$, et al. Predicting smoking cessation. Who will quit with and without the nicotine patch. JAMA 1994; 271: 589-94.

13. Szychta W, Majstrak F, Opolski G, Filipiak KJ. Change in the clinical profile of patients referred for coronary artery bypass grafting from 2004 to 2008. Trends in a single-centre study. Kardiol Pol 2015; 73: 493-501.

14. Szychta W, Majstrak F, Opolski G, Filipiak KJ. Trends in pharmacological therapy of patients referred for coronary artery bypass grafting between 2004 and 2008 - a single center study. Kardiol Pol 2015; 73: 1317-26.

15. Knapik P, Nadziakiewicz P, Urbanska E, et al. Cardiopulmonary bypass increases postoperative glycemia and insulin consumption after coronary surgery. Ann Thorac Surg 2009; 87: 1859-65.

16. Frioud A, Comte-Perret S, Nguyen S, et al. Blood glucose level on postoperative day 1 is predictive of adverse outcomes after cardiovascular surgery. Diabetes Metab 2010; 36: 36-42.

17. Wiener R, Wiener D, Larson R. Benefits and risks of tight glucose control in critically ill adults: a meta-analysis. JAMA 2008; 300: 933-44.

18. Lazar HL, McDonnell M, Chipkin SR, et al. The Society of Thoracic Surgeons practice guideline series: blood glucose management during adult cardiac surgery. Ann Thorac Surg 2009; 87: 663-9.

19. Filsoufi F, Rahmanian PB, Castillo JG, et al. Diabetes is not a risk factor for hospital mortality following contemporary coronary artery bypass grafting. Interact Cardiovasc Thorac Surg 2007; 6: 753-8.

20. Loop F, Lytle B, Cosgrove D, et al. Influence of the internal-mammary-artery graft on 10-year survival and other cardiac events. N Engl J Med 1986; 314: 1-6.

21. Holzmann MJ, Rathsman B, Eliasson B, et al. Long-term prognosis in patients with type 1 and 2 diabetes mellitus after coronary artery bypass grafting. J Am Coll Cardiol 2015; 65: 1644-52.

22. Montalescot G, Sechtem U, Achenbach S, et al. 2013 ESC guidelines on the management of stable coronary artery disease: the Task Force on the management of stable coronary artery disease of the European Society of Cardiology. Eur Heart J 2013; 34: 2949-3003. 